\title{
Self-contained Web Components through Serverless Computing
}

\author{
Markus Ast \\ Chemnitz University of Technology \\ Chemnitz, Saxony \\ markus.ast@informatik.tu-chemnitz.de
}

\author{
Martin Gaedke \\ Chemnitz University of Technology \\ Chemnitz, Saxony \\ martin.gaedke@informatik.tu-chemnitz.de
}

\begin{abstract}
Web Components are an essential building block for modularizing large and complex web applications into smaller pieces. Due to the web's nature, functionality encapsulated into Web Components consist of presentation and business logic. The former executes inside the user agent and the latter on the server-side. Many Web Components are not self-contained because they require to integrate their business logic into your backend or to use externally hosted third-party services. In this paper, we describe an approach of how to utilize Serverless Computing to enable self-contained web components by deploying Web Component business logic as cloud-hosted functions.
\end{abstract}

\section{CCS CONCEPTS}

- Information systems $\rightarrow$ Web interfaces; Web services; Web applications; $\bullet$ Computer systems organization $\rightarrow$ Cloud computing; • Human-centered computing $\rightarrow$ User interface programming;

\section{KEYWORDS}

Serverless Computing, Function as a Service, Web Components, Web Services, Micro Services, End-User Development

\section{ACM Reference Format:}

Markus Ast and Martin Gaedke. 2017. Self-contained Web Components through Serverless Computing. In WoSC'17: WoSC'17: Workshop on Serverless Computing, December 11-15, 2017, Las Vegas, NV, USA. ACM, New York, NY, USA, 6 pages. https://doi.org/10.1145/3154847.3154849

\section{INTRODUCTION}

More and more of today's software is modularized into smaller self-contained pieces. In web engineering, those pieces are often referred to as modules or microservices on the server-side, and as components on the client-side. To illustrate the differences between them, consider a upvote functionality that should be integrated into a blog. Users can click the upvote button which increases a counter showing how many people have positively rated a certain article. Using the recent Web Components W3C standards, the upvote button component can be integrated into the blog by simply

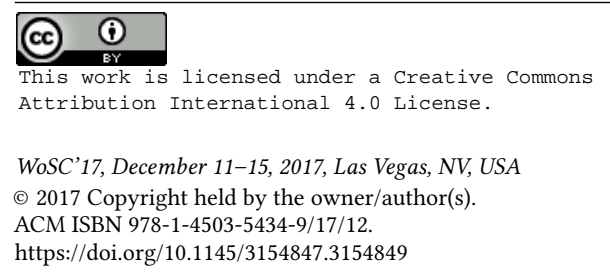

loading the corresponding script file and by adding its custom HTML tag, as shown in Listing 1:

\section{Listing 1: Integrating a Web Component into a Website}

<script type="module" src="upvote-button.js">

$</$ script $>$

<upvote-button $></$ upvote-button $>$

The JavaScript loaded with upvote-button.js contains the HTML, CSS and presentation logic of the button. However, one important part is missing: the business logic for restricting votes to one vote per IP address and for saving votes into a persistent data storage. Since such business logic lives on the server, it is not already contained in the upvote-component. It rather relies on integrating the functionality from scratch into an existing backend, on using a dedicated module that can be integrated into an existing backend, or the component can be built against a microservice. Latter can be provided as a self-hosted service or as an external third-party hosted service. Unfortunately, only the third-party hosted microservice solution provides a truly self-contained upvote-component, i.e., a web component that comes with both front- and on-premise backend logic integrated and works out of the box. This is, however, often undesired because of reasons like:

- it locks you into the specific service which might shut down in the future (because it is being bought, abandoned or goes bankrupt),

- composing applications from many small components would lead to having to manage dozens of external services with their respective billing and

- it takes the underlying data out of your control.

In this paper, we first explore in Section 2 the possibility of improving on these constraints by employing Serverless Computing and consequentially enabling the creation Serverless Web Components, i.e., of web components that are truly self-contained. After that we show in Section 2.3 that having truly self-contained web components can:

- increase the development speed of new applications,

- decreases the costs of development of new applications,

- enables global scale but still decreases ongoing costs,

- acts as an enabler for powerful end-user development platforms.

Finally, we show an example in Section 3 and dive into related work in Section 4 before we give a conclusion and an outlook on future work in Section 5. 


\section{APPROACH}

Our goal is to enable truly self-contained web components âĂŞ web components that work out of the box. That is, it does not only include its UI and corresponding presentation logic but is also include its server-side business logic. We envision this being possible by employing the sound approach of Serverless Computing.

Serverless Web Components have an integrated deployment workflow. When a website creator first adds a Serverless Web Component to their website, instead of showing the component, a deploy button gets displayed. Once clicked, this button redirects to the cloud provider of choice and asks for authorization to deploy the FaaS that is attached to the component. Once authorized, the user gets redirected back to their site. With the function deployed, the deploy button is not displayed anymore. Instead, the actual UI of the component is shown and functioning against the previously deployed function. The process is illustrated in Figure 1.

The whole process should work without getting locked into a specific cloud provider. Once, for the first deployment, the process includes the initial account creation at a compatible cloud provider. There are then two approaches of how to specify the website's cloud provider. Either with a global element that propagates the configuration to all compatible components or by defining it for each component separately, as shown in Listing 2.

Listing 2: Specify a Page's/Component's FaaS Endpoint <serverless-config endpoint="https://example.org/faas

$\hookrightarrow$ /projectX" $></$ serverless-config $>$

$<$ upvote-button $><$ /upvote-button $>$

$<!--$ OR -->

<upvote-button endpoint="https://example.org/faas/

$\hookrightarrow$ projectX" $><$ /upvote-button $>$

\subsection{Deployment}

In our goal, a cloud provider would allow deploying functions through a simple link. The link would take a source Git repository (or a source of another kind) as an argument. It would then show an authorization page for the deployment of this one function. This page could contain some additional cloud provider-specific fields like the project the function should be deployed to. Once authorized, the cloud provider fetches the function from the provided source repository and deploys it accordingly. The sequence of this deployment process is shown in Figure 2.

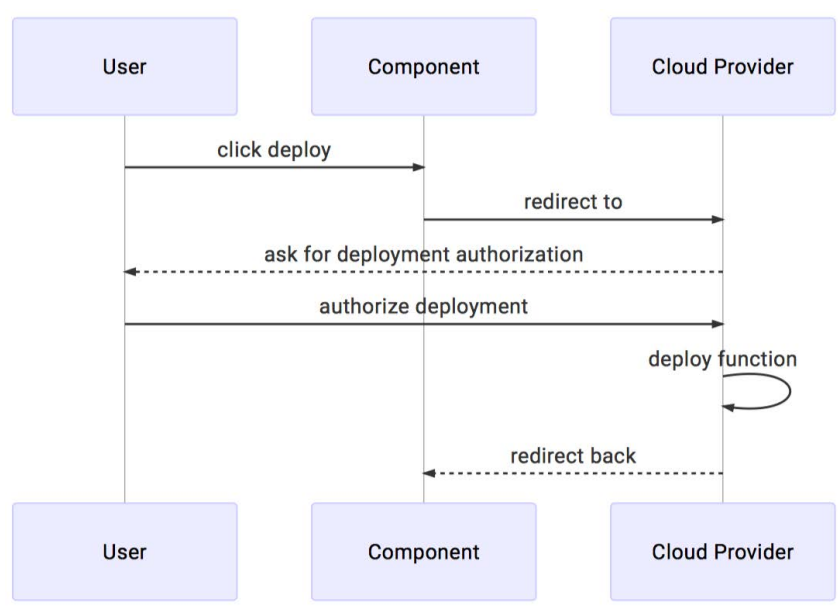

Figure 2: Sequence Diagram of a User deploying a Web Component directly into their Cloud Provider

An important part is that this authorization is a one-time privilege for the creation of one single function and not a token that grants access for the whole functions management. This constraint is crucial for the deployments of non-open source functions. Deployments of non-open source functions would work through the function's vendor, which deploys the function on behalf of the user. In such a scenario, you wouldn't want to grant the provider full access to all your functions. Instead, you just want to grant privileges to deploy this one single function only once. This scenario is illustrated in the sequence diagram in Figure 3. In this scenario, the component redirects to its vendor for deployment. The vendor itself redirects further to the cloud provider adding details about itself to the deployment request. Subsequently, the user is asked to authorize this deployment. Once authorized, the cloud provider redirects back to the Component Vendor providing a one-time deployment token, which the Component Vendor uses to deploy the function. This scenario of deploying proprietary functions would, however, require the cloud providers to prevent the user from downloading the corresponding deployed functions. Downloading the source of deployed functions currently possible in most FaaS cloud providers.

Unfortunately, the described link-based one-time deployment is not available, yet. The alternative solution is to provide an intermediate service, which we choose for our proof of concept. The service

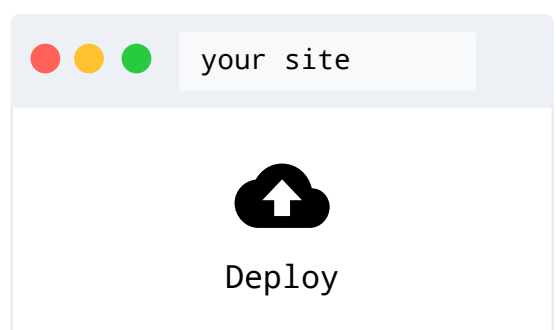

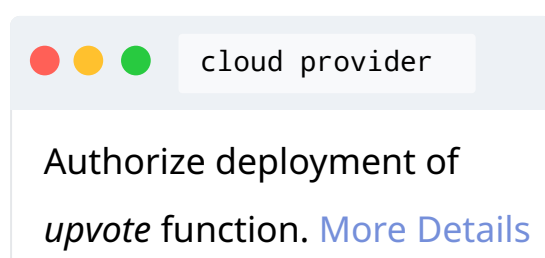

Authorize

\section{your site}

\section{I6 42}

Figure 1: Integrated Deployment Process of Serverless Web Components 


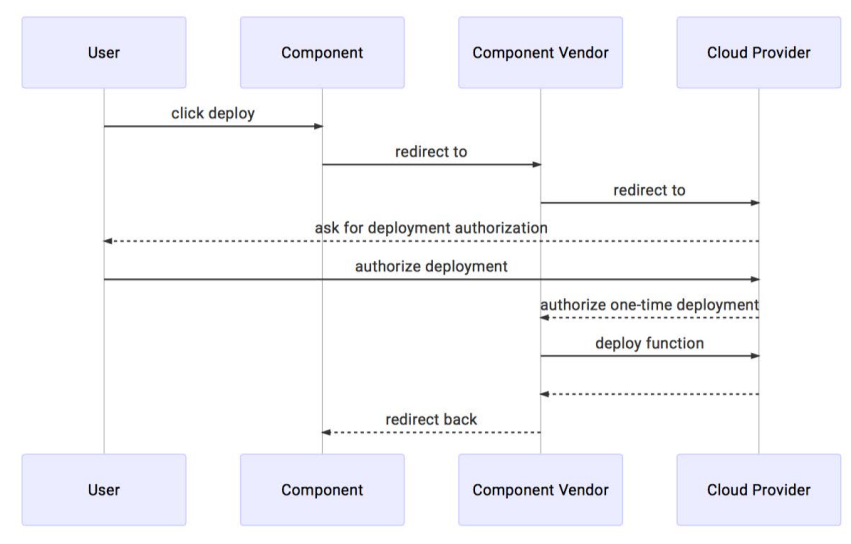

Figure 3: Sequence Diagram of a User deploying a proprietary Function through the Function's Vendor into the User's Cloud Provider

is authorized by the cloud provider of choice and offers deployment as described above, with the difference being deployment requests are authorized and executed by the intermediate service instead of by the Cloud Provider directly, as shown in Figure 4.

While this intermediate service comes with the drawback of having to grant full access to managing a user's cloud functions, it also has advantages. Some advantages we want to highlight are:

- Since it is a single hub where functions are deployed from, it can provide a user-friendly overview over all functions that are deployed for a certain host.

- As a single hub, it can be extended to detect function-source changes in a Git repository and provide the user with a one-click update functionality.

- It can wrap deployed function with an additional layer of functionality and security.

The latter could also be added by a function's developer manually but would increase the boilerplate code. The functionality, which is required to execute AJAX calls (HTTP requests from JavaScript) against the service, is to add proper Cross-Origin Resource Sharing (CORS) HTTP headers. By only enabling CORS for the deploying host of a function, it ensures that the corresponding component cannot be integrated into other websites. This protects the user from others who might try to use functions that others pay for. An exemplary CORS layer is shown in Listing 3.

Listing 3: Function wrapper that sets CORS-header for incoming HTTP requests to restrict function calls (through AJAX calls) to the owner's host.

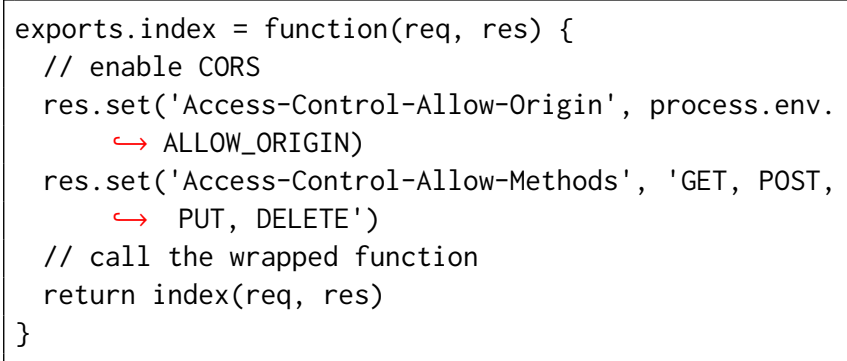

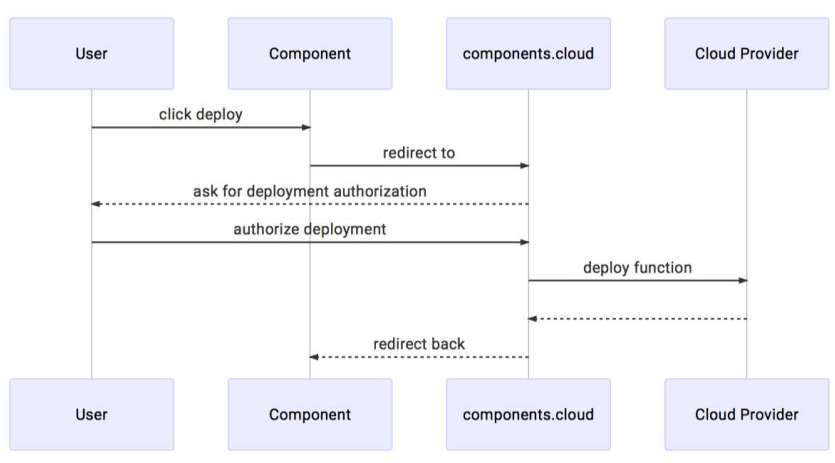

Figure 4: Sequence Diagram of a User using an intermediate Service (components.cloud) to deploy a Function to their Cloud Provider

A proof of concept service is available at http://components. cloud.

\subsection{Deployable Web Components}

Since the deployment button for Serverless Web Components is a recurring pattern, it is something every component developer shouldn't be required to implement again and again. Fortunately, JavaScript's prototypal inheritance can be used to inherit new Serverless Web Components from a base component, which automatically provides the deployment process. Listing 4 shows, what developing a Serverless Web Component would then look like ? the newly created UpVoteElement inherits from the base component ServerlessComponent, while ServerlessComponent inherits from HTMLElement). Additionally, when instantiating the component, it would pass options to ServerlessComponent, like the Git repository where the component's function source can be retrieved from.

Listing 4: Inherit the Deployment Process from a base Component

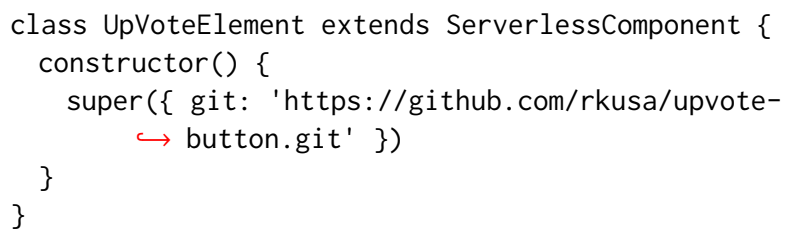

The component must also be able to detect whether it has already been deployed or not. There are two possible approaches for detecting the deployment state. First, the ServerlessComponent could explicitly query, once for a session, whether it has been deployed, or not. Second, instead of actively querying the deployment state, the state could also be detected passively. This is possible for use cases where the component queries a function when being loaded anyway, to retrieve its initial data. To reduce the number of requests, this for the component mandatory request can be used to derive the deployment state.

The idea is, that when enabling a user to host a service through their account of the cloud provider of their choice, it prevents a user from being locked into a specific service that might shut down in 
the future. It additionally allows the user to have all the data from all services in one, from the user accessible, place. However, due to differences in cloud providers in terms of supported programming languages, differences of how entry points are defined and different APIs for other services like databases and file storage [6], this is not completely achieved, yet. Achieving such a compatibility between cloud providers is part of future work (Section 5).

\subsection{Why Serverless Computing?}

While it is possible to argue that described approach would also work with Infrastructure as a Service (IaaS) or Platform as a Service (PaaS), we think that FaaS is the better fit for Serverless Web Components due to the following reasons. At first, since the approaches targets end-user development in the long term, not having to manage virtual servers, capacity, utilization, availability and fault tolerance [2] is essential because it makes the approach accessible for a significant bigger target-group.

Another important factor are ongoing costs. Having to deploy a microservice to one server/one node quickly increase monthly costs. According to [11], by exclusively deploying microservices on AWS Lambda, infrastructure costs can be reduced by up to $77.08 \%$. There is, of course, a point, where IaaS becomes cheaper than solely relying on FaaS. According to [16], an AWS m4.large instance (2 vCPUs and 8GB RAM) "would need to be running 82 requests per second, every second of every day, before it is more cost-effective than running the same workload on Lambda". However, this is a workload that isn't expected in most Server Web Components use cases. For a normal lightweight and low-traffic website, [16] concluded that using AWS Lambda is $1 / 10$ th cheaper than using t2.nano, the smallest AWS EC2 instance. In Serverless Computing you only pay resources that are actually used.

Furthermore, when thinking about global scale and about reducing the latency for users all over the globe, using IaaS or PaaS requires creating additional servers/nodes per server center location. For three server center locations, this increase already triples the costs. In contrast, deploying FaaS at global scale does not come with extra costs, because you are still only paying the function calls. Just the costs per function call might differ slightly between certain locations.
Besides the hosting aspect, since the user is not required to integrate the business logic needed for integrating components into their own backend, the development speed is significantly reduced [2]. Consequentially, development costs are reduced as well. In the end, the time to market reduced [2] for new applications or new features.

\section{EXAMPLE}

To better illustrate the approach of this paper, we will take a look at an example Serverless Web Component. There are a lot of possible use-cases, even complex ones like eCommerce functionality. However, for the sake of illustration, we will use the simple example of an upvote component. Suppose we have already set up our cloud provider of choice and add the upvote component to our site, as shown in Listing 5.

Listing 5: HTML tags for adding the Example UpVote Component to a Website

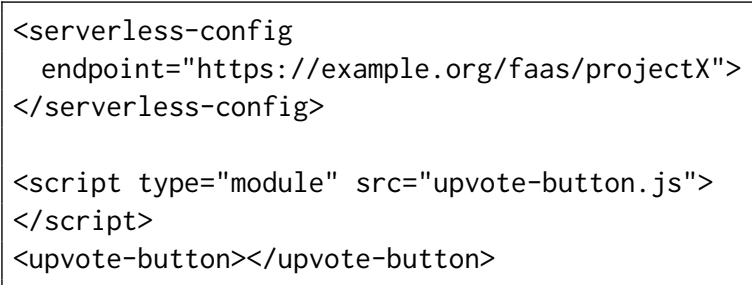

When opening the page where the component is included, instead of the upvote button, a deploy button is shown, as seen in in the leftmost screenshot in Figure 5. This is of course only the case as long as the component's function is not yet deployed. Once deployed, the component works regularly (and is shown as seen in the rightmost screenshot in Figure 5).

As of the Web Components standard, the custom HTML tag of upvote-button can be defined as follows (code is shortened for readability). A new prototype named UpVoteElement is created which extends from the Server lessComponent prototype. Latter adds the deployment process. When being loaded the upvote component retrieves the current count and when being clicked, the component increases (or decreases) the current count. The component is registered as the custom HTML tag upvote-button.
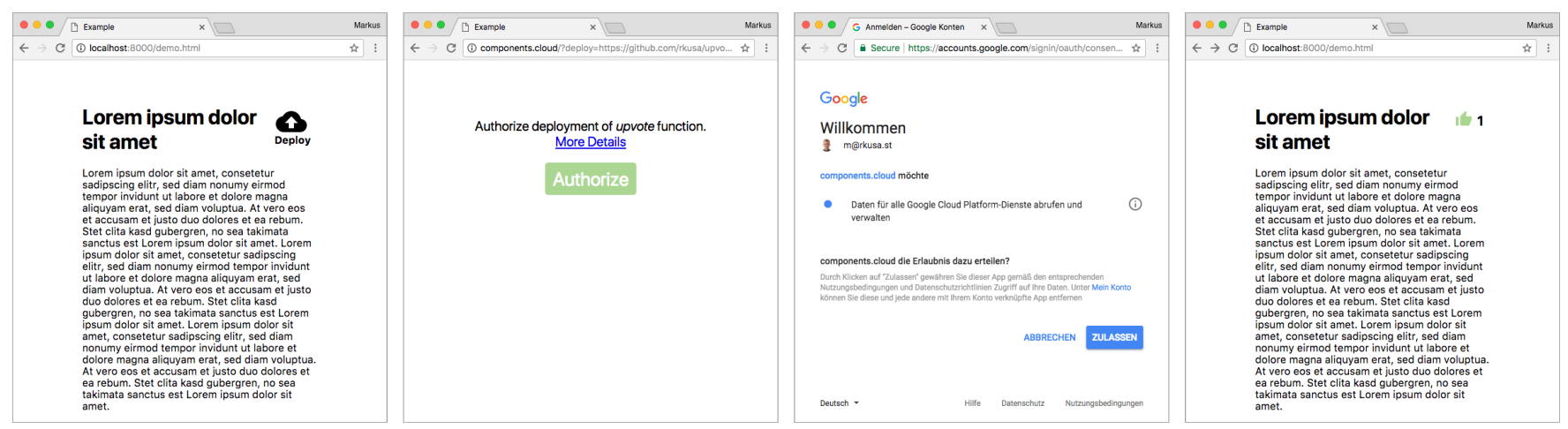

Figure 5: Screenshot of Vote example Web Component. From left to right: Deploy Link, One-time deployment authorization, Authorize the intermediate Service for a Cloud Provider, the deployed and working Serverless Web Component 


\section{Listing 6: Source of UpVote Web Component}

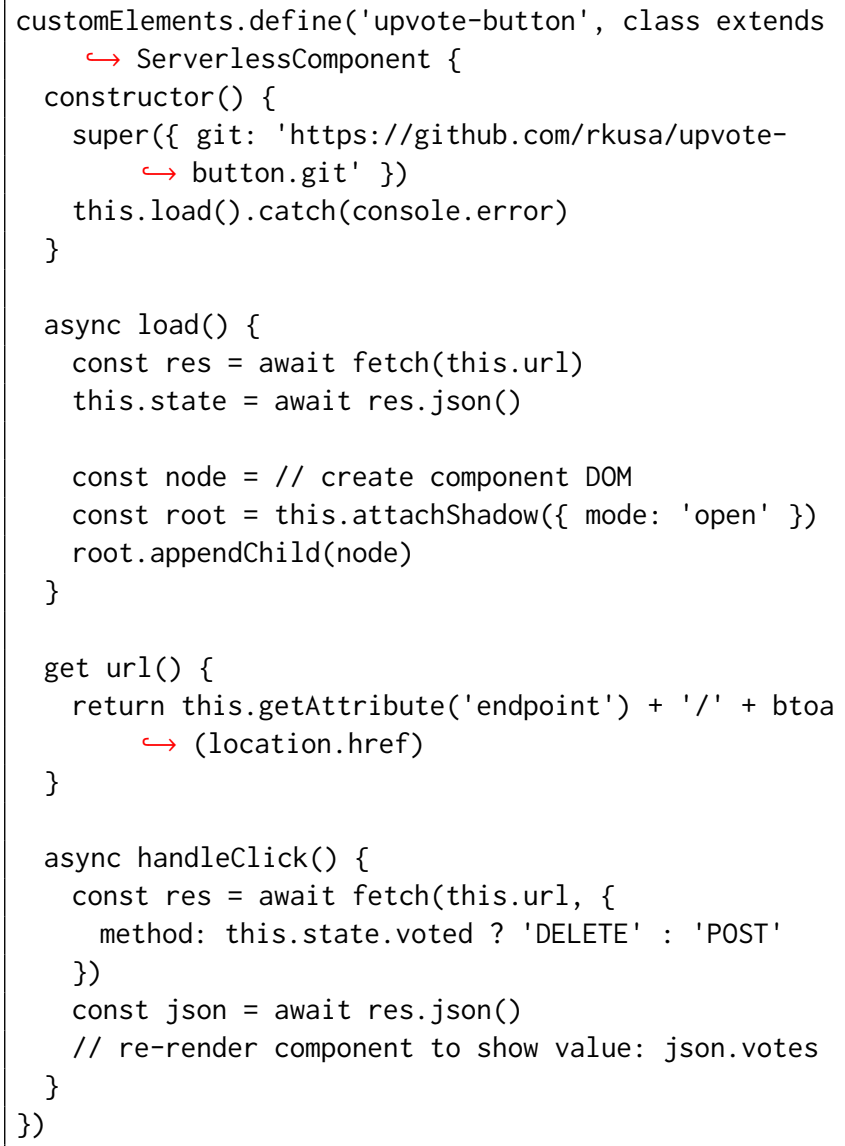

Internally the upvote button works as shown in Figure 6. When loaded, the component determines a unique identifier for the page where it is included. The component uses the base64-encoded URL of the current page as the unique identifier (: uid in the REST API) for voting. This allows to scope votes to a specific page. This unique identifier is used to load the current vote count, which is then rendered in the component's UI. When being clicked, the component sends a request to its function, which determines whether the vote is unique (based on the voter's IP address) and updates the vote count accordingly. The component receives the new vote count as a response and reflects changes in its UI.

The component's backend is a set of functions that can be triggered with HTTP requests, ie. it is basically a RESTful API. It provides the following routes:

- GET /upvote/:uid Get current vote count for the unique identifier : uid

- POST /upvote/:uid Vote for the unique identifier : uid

- DELETE /upvote/:uid Remove vote for the unique identifier : uid

The POST route would only allow one vote per IP address, and if there is any vote for a calling IP address, a call to the DELETE route will remove it. While only filtering by IP address is not the most sophisticated way of ensuring a single vote per person in an account-less system, it is sufficient for the sake of this example.

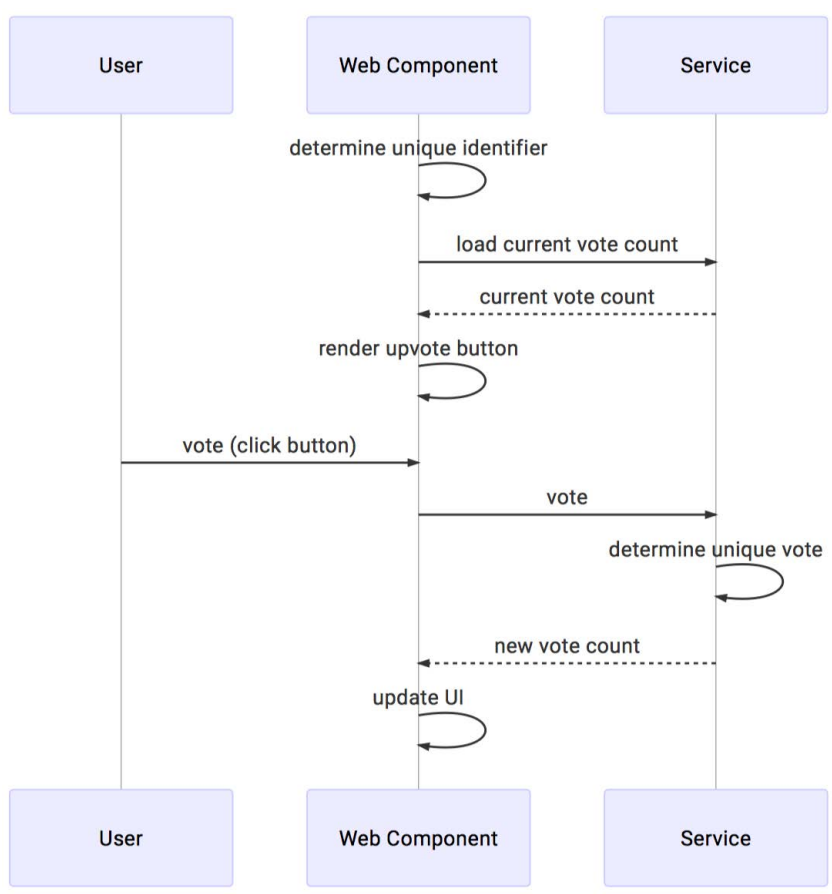

Figure 6: Sequence Diagram of how the example UpVote Component works

\section{RELATED WORK}

The approach of Serverless Web Components mainly consists of the technologies Web Components and Serverless Computing, which we will shortly examine in this section.

\subsection{Web Components}

Building big complex things out of smaller less complex things is a fundamental approach of modern software engineering[10]. It enables encapsulation, reusability, interoperability, reduction of complexity and better testability. Such small and less complex things are commonly referred to as Components. Components, as building blocks for applications, encapsulate reasonable small and self-contained functionality.

For the web, more and more libraries for dissecting large complex applications into smaller parts evolved in the last decay. For instance, jQuery Plugins ${ }^{1}$, or later movements like W3C widgets[12] or the Dojo toolkit ${ }^{2}$. The current and future trend for web development shows a rapid increase in usage of component-based approaches, even though they have evolved into more sophisticated libraries. While $\mathrm{W} 3 \mathrm{C}$ widgets were one of the major players for so-called Mashups[3-5] and [5] confirm that the trend is moving towards the Web Components standard.

Four of the Top10 JavaScript projects hosted on the biggest Open Source platform Github ${ }^{3}$ are frameworks: React ${ }^{4}$, React Native ${ }^{5}$

\footnotetext{
${ }^{1}$ https://plugins.jquery.com

${ }^{2}$ https://dojotoolkit.org

${ }^{3}$ https://git.io/vFkaO (Accessed: 31.09.2017)

${ }^{4}$ https://facebook.github.io/react/

${ }^{5}$ https://facebook.github.io/react-native/
} 
(both build by Facebook), Vue. js ${ }^{6}$ and Angular ${ }^{7}$ (build by Google). All of those four frameworks are component-based. Additionally, almost all of the currently most used JavaScript frameworks are component-based as well, e.g., Polymer ${ }^{8}$ (build by Google) and glimmer ${ }^{9}$. However, all those frameworks have their own user base and lack interoperability (Angular has a Web Component interoperability extension).

The Web Components standard therefore brings components into the platform itself making it part of the web technology stack instead of relying on libraries. It consists of four main specifications: Custom Elements[13], Shadow DOM[15], HTML Imports[14] and HTML Templates[1] with their respective role being the definition of custom HTML tags, the encapsulation of the component from other components, the loading of the components and the re-use of HTML fragments.

While the in this paper suggested approach could be implemented on top of all mentioned component-based frontend frameworks, we choose to focus on Web Components because it is a standard that is built right into the platform and is expected to be the building block of future frameworks, making it future-proof.

\subsection{Serverless Computing}

Function as a Service (FaaS) and Serverless Computing are a "cloudnative platform for short-running, stateless computation and eventdriven applications which scales up and down instantly and automatically and charges for actual usage at a millisecond granularity." [6] Serverless Computing is, after Grid, Infrastructure as a Service and Platform as Service, Serverless Computing, the next evolution of cloud computing[2]. As of [8], it becomes increasingly relevant as the future cloud service abstraction. With Serverless Computing, it is about writing stateless and event-based [9] server-side code as so-called Functions as the unit of deployment and scale[?], without having to manage any server infrastructure [2]. Functions are triggered by events, like HTTP calls to a certain URL, timers or events like a newly created file in a data storage.

Some of the currently available Serverless clouds are IBM OpenWhisk $^{10}$, Google Cloud Functions ${ }^{11}$, AWS Lambda ${ }^{12}$ and Azure Functions ${ }^{13}$.

\section{CONCLUSION AND FUTURE WORK}

In this paper, we demonstrated how Serverless Computing and Web Components could be combined to create truly self-contained Serverless Web Components. Such components are easy to integrate into existing websites and can be used to add additional functionality to websites quickly. It thereby reduces the time required to add functionality to a website and simultaneously reduces the costs for global scale.

There are still some weak points and further challenges. First, the demonstrated solution cannot be integrated into existing cloud

\footnotetext{
${ }^{6} \mathrm{https} / /$ vuejs.org/

${ }^{7} \mathrm{https} / / /$ angular.io/

${ }^{8}$ https://www.polymer-project.org/

${ }^{9} \mathrm{https}: / /$ glimmerjs.com/

${ }^{10} \mathrm{https} / / /$ www.ibm.com/cloud-computing/bluemix/openwhisk

${ }^{11}$ https://cloud.google.com/functions/

${ }^{12} \mathrm{https}$ ://aws.amazon.com/de/lambda/

${ }^{13}$ https://azure.microsoft.com/de-de/services/functions/
}

providers yet. It is therefore required to use an intermediate service with management privileges to a user's cloud provider. This might concur with a user's privacy requirements. Additionally, since the target group of the approach are also non-developer users, the whole user experience for setting up the cloud is not yet as good as it could be. Furthermore, FaaS cloud providers still have a lock-in effect, due to incompatible APIs[6]. That is, we would like the see the adoption of the following points in the future: a) Support for simple one-time deployments, b) Standard FaaS API (and compatible APIs for other related services) and c) Better UX for setting up a FaaS cloud account.

Besides these points, which could be achieved by providing middleware, we will focus on improving the easiness and user experience of the deployment and management of Serverless Web Components, on integrating this approach into end-user development platforms and on using this solution as a foundation for a WebComposition [7] approach based on Serverless Computing.

\section{ACKNOWLEDGMENT}

The authors gratefully acknowledge funding by the DFG (GRK 1780/1).

\section{REFERENCES}

[1] 2017. HTML Standard. (Aug. 2017). https://html.spec.whatwg.org/multipage/ webappapis.html\#scripting

[2] Roger Barga. 2017. Serverless Computing: Patterns and Road Ahead. (2017). http://www.serverlesscomputing.org/wosc17/presentations/ barga-keynote-serverless.pdf

[3] S Casteleyn, P Dolog, and C Pautasso. 2016. Current Trends in Web Engineering. Springer (2016).

[4] Olexiy Chudnovskyy, Heng Chang, Tobias Nestler, Martin Gaedke, Florian Daniel, José Ignacio Fernández-Villamor, Vadim Chepegin, José Angel Fornas, Scott Wilson, and Christoph Kögler. 2012. End-user-oriented telco mashups: the OMELETTE approach. In the 21st international conference companion. ACM Press, New York, New York, USA, 235.

[5] Florian Daniel and Martin Gaedke. 2016. ICWE 2016 Rapid Mashup Challenge: Introduction. In Rapid Mashup Development Tools. Springer, Cham, Cham, 1-9.

[6] Geoffrey Charles Fox, Vatche Ishakian, Vinod Muthusamy, and Aleksander Slominski. 2017. Status of Serverless Computing and Function- as-a-Service(FaaS) in Industry and Research. (Sept. 2017), 1-23.

[7] Martin Gaedke and Guntram Gräf. 2001. Development and Evolution of WebApplications Using the WebComposition Process Model. Web Engineering 2016, Chapter 8 (2001), 58-76.

[8] Craig Lowery. 2016. Emerging Technology Analysis: Serverless Computing and Function Platform as a Service. (Sept. 2016).

[9] Garrett McGrath, Paul R Brenner, Brenden Judson, Jared Short, and Stephen Ennis. 2016. Cloud Event Programming Paradigms. (June 2016), 1-7.

[10] Rainer Niekamp. 2006. Software Component Architecture. (April 2006), 1-59.

[11] Mario Villamizar, Oscar Garces, Lina Ochoa, Harold Castro, Lorena Salamanca, Mauricio Verano, Rubby Casallas, Santiago Gil, Carlos Valencia, Angee Zambrano, and Mery Lang. 2016. Infrastructure Cost Comparison of Running Web Applications in the Cloud Using AWS Lambda and Monolithic and Microservice Architectures. In 2016 16th IEEE/ACM International Symposium on Cluster, Cloud and Grid Computing (CCGrid). IEEE, 179-182.

[12] W3C Working Group. 2012. Packaged Web Apps (Widgets) - Packaging and XML Configuration (Second Edition). (Nov. 2012). https://www.w3.org/TR/widgets/

[13] W3C Working Group. 2017. Custom Elements. (Feb. 2017). https://w3c.github. io/webcomponents/spec/custom/

[14] W3C Working Group. 2017. HTML Imports. (Feb. 2017). https://w3c.github.io/ webcomponents/spec/imports/

[15] W3C Working Group. 2017. Shadow DOM. (Feb. 2017). https://w3c.github.io/ webcomponents/spec/shadow/

[16] Andy Warzon. 2016. AWS Lambda Pricing in Context - A Comparison to EC2. (Aug. 2016). https://www.trek10.com/blog/lambda-cost/ 